Revue d'histoire de l'Amérique française

REVUE D.HISTOIRE DE L'AMÉRIQUE FRANÇAISE

\title{
Naissance d'une élite : les médecins dans la société saguenayenne (1850-1940)
}

\section{Gérard Bouchard}

Volume 49, numéro 4, printemps 1996

URI : https://id.erudit.org/iderudit/305463ar

DOI : https://doi.org/10.7202/305463ar

Aller au sommaire du numéro

Éditeur(s)

Institut d'histoire de l'Amérique française

ISSN

0035-2357 (imprimé)

1492-1383 (numérique)

Découvrir la revue

Citer cet article

Bouchard, G. (1996). Naissance d'une élite : les médecins dans la société saguenayenne (1850-1940). Revue d'histoire de l'Amérique française, 49(4), 521-549. https://doi.org/10.7202/305463ar
Résumé de l'article

Cet essai reconstitue les efforts persistants et fructueux des médecins (et chirurgiens) du Saguenay pour se hisser au rang des élites régionales à partir de la seconde moitié du XIX ${ }^{\mathrm{e}}$ siècle. Au cours des premières décennies du peuplement, le corps médical se trouvait confronté à un double défi. Il devait d'abord structurer sa profession et ensuite la faire reconnaître dans une société largement sollicitée par les tâches de la colonisation et imprégnée de ses modes de vie. Ce double défi exigeait une triple accréditation, sur les plans scientifique, social et culturel. Selon la thèse défendue par l'auteur, la promotion sociale du médecin à l'époque considérée (1850-1940) s'est appuyée davantage sur des stratégies culturelles et sociopolitiques que sur des privilèges ou avantages économiques et strictement scientifiques.
Tous droits réservés @ Institut d'histoire de l'Amérique française, 1996
Ce document est protégé par la loi sur le droit d'auteur. L'utilisation des services d'Érudit (y compris la reproduction) est assujettie à sa politique d'utilisation que vous pouvez consulter en ligne.

https://apropos.erudit.org/fr/usagers/politique-dutilisation/ 


\title{
NAISSANCE D'UNE ÉLITE: LES MÉDECINS DANS LA SOCIÉTÉ SAGUENAYENNE (1850-1940) ${ }^{1}$
}

\author{
GÉRARD BOUCHARD \\ Institut interuniversitaire de recherches sur les populations \\ Université du Québec à Chicoutimi
}

\section{RÉSUMÉ}

Cet essai reconstitue les efforts persistants et fructueux des médecins (et chirurgiens) du Saguenay pour se hisser au rang des élites régionales à partir de la seconde moitié du XIX ${ }^{c}$ siècle. Au cours des premières décennies du peuplement, le corps médical se trouvait confronté à un double défi. Il devait d'abord structurer sa profession et ensuite la faire reconnaître dans une société largement sollicitée par les tâches de la colonisation et imprégnée de ses modes de vie. Ce double défi exigeait une triple accréditation, sur les plans scientifique, social et culturel. Selon la thèse défendue par l'auteur, la promotion sociale du médecin à l'époque considérée (18501940) s'est appuyée davantage sur des stratégies culturelles et sociopolitiques que sur des privilèges ou avantages économiques et strictement scientifiques.

\section{ABSTRACT}

This paper analyses the long-lasting and successful attempt by the Saguenay physicians (and surgeons) to climb the social ladder in this region and to secure for themselves a place among the elites. During the first decades of the settlement (in the second half of the 19th century), the medical body was faced with a double challenge. It has to, first, structure its profession and, second, fight for its recognition by the society as a whole. The latter involved an acceptation, by this overwhelmingly rural population, at three levels: scientific, social, and cultural. According to the thesis set forth by the author, this could be mainly achieved through socio-political and cultural

1. Notre enquête a pu être réalisée grâce à l'appui financier du Conseil de recherches en sciences humaines du Canada, du Fonds FCAR (du gouvernement du Québec) et de l'Université du Québec à Chicoutimi. Nous remercions Judith Goulet et Jeannette Larouche (traitement, relecture du texte) de même que Marc Saint-Hilaire, Francine Saillant, Jacques Bernier, Normand Perron, Guildo Rousseau et Denis Goulet qui ont bien voulu commenter une version précédente du manuscrit. Johanne Daigle nous a également transmis d'utiles informations. L'auteur n'a pas cependant donné suite à toutes les suggestions qui lui ont été faites et il demeure entièrement responsable des orientations proposées.

RHAF, vol. 49, $\mathrm{n}^{\circ}$ 4, printemps 1996 
strategies. As a corollary, it appears that wealth and scientific knowledge, while undoubtedly contributing to the social promotion of the medical body, did not act as primary factors.

\section{1 - ESSOR D'UNE ÉLITE DANS UNE COLLECTIVITÉ NEUVE}

Cet essai reconstitue les efforts des médecins (et chirurgiens) du Saguenay pour se hisser au rang des élites régionales à partir de la seconde moitié du $\mathrm{XIX}^{\mathrm{e}}$ siècle. L'environnement géographique et social de cette difficile ascension était celui d'une collectivité neuve en formation. C'est à la fin de la décennie 1830 qu'a commencé la colonisation du Saguenay, dont la population s'élevait à 6000 habitants en 1851, puis à 37000 en 1901 et, enfin, à 198000 en $1951^{2}$. Un réseau urbain s'implanta assez rapidement durant le premier tiers du $\mathrm{XX}^{\mathrm{e}}$ siècle, mais il était constitué de toutes petites villes à croissance lente; en 1931, 80\% de la population vivait encore dans des agglomérations de moins de 5000 habitants, même si la région comprenait alors une dizaine de villes ${ }^{3}$. La période couverte par notre enquête s'étend de l'arrivée des premiers médecins dans les années 1840 à la fin de la colonisation du territoire saguenayen.

L'objectif poursuivi est de montrer comment les membres de la communauté médicale ont réussi leur insertion dans cette société en formation et par quelles stratégies ils sont parvenus à s'y constituer comme une élite spécifique, reconnue par l'ensemble de la société. Il nous faudra donc passer en revue la croissance et l'implantation du corps médical, les modalités d'accréditation de son savoir, les résistances et les obstacles auxquels il a été confronté, son organisation corporative, la reconnaissance sociale et culturelle qui l'a finalement et durablement installé dans la notabilitét. Sans qu'il n'y paraisse trop

2. Christian Pouyez, Yolande Lavoie, Gérard Bouchard, Raymond Roy et al., Les Saguenayens. Introduction à l'histoire des populations du Saguenay, XVIe-XXe siècles (Québec, Presses de l'Université du Québec, 1983), 386 p., tableau 6.2. (Prix Lionel-Groulx, de l'Institut d'histoire de l'Amérique française. Certificat de Mérite de la Société Historique du Canada).

3. En 1931, Chicoutimi (11 877 habitants) et Jonquière (9 448 habitants) étaient les plus grosses villes. Gérard Bouchard, «Le peuplement blanc», C. Pouyez, Y. Lavoie, G. Bouchard, R. Roy et al., Les Saguenayens. Introduction à l'histoire des populations du Saguenay, XVIeXXe siècles (Québec, Presses de l'Université du Québec, 1983), 125-180.

4. Nous avons déjà traité ailleurs le cas des élites socio-économiques et des élites religieuses (ou cléricales) dans cette région. Gérard Bouchard, Yves Otis, France Markowski, «Les notables du Saguenay au $\mathrm{XX}^{\mathrm{e}}$ siècle à travers deux corpus biographiques», Revue d'histoire de l'Amérique française, 39,1 (été 1985): 3-23; Gérard Bouchard, Raymond Roy, Pierre Jacques, «La composition des communautés de religieuses au Saguenay (1882-1947)», La Société canadienne d'histoire de l'Église catholique, Sessions d'étude, 55 (1988): 87-117; Gérard Bouchard, «Origines géographiques et sociales du personnel religieux dans la région du Saguenay (1882-1947)", à paraître dans Histoire sociale/Social History, 28,55 (mai 1995); Gérard Bouchard, «Familles à prêtres? Familles à sœurs? Parenté et recrutement religieux au Saguenay 
(le sujet n'a jamais été au cœur des débats scientifiques), l'historiographie québécoise compte un nombre assez élevé de travaux souvent modestes, il est vrai - sur l'histoire de la médecine et des médecins. Plusieurs de ces études sont parues dans des revues spécialisées comme l'Union médicale du Canada et le Laval médical, ou le Bulletin des recherches historiques et les Cahiers des Dix. Certaines sont mieux connues, celle de George Weisz ${ }^{5}$, par exemple, ou de François Rousseau sur l'Hôtel-Dieu de Québec (deux tomes parus), ou encore les ouvrages-synthèses de Jacques Bernier sur la période 1788-1909, puis ceux de Denis Goulet et d'André Paradis sur la période $1639-1939^{6}$. Parmi les recherches pionnières, mentionnons aussi la contribution de Fernand Ouellet ${ }^{7}$ qui, le premier, a fourni un aperçu quantitatif de la profession médicale pour le premier tiers du $\mathrm{XIX}^{\mathrm{e}}$ siècle. Les sociologues se sont aussi intéressés au sujet sous l'angle de la professionnalisation ${ }^{8}$.

Cette dimension est au cœur du présent essai, dont la problématique est toutefois plus large. Durant les premières décennies du peuplement, les médecins du Saguenay se trouvaient confrontés à un double défi. Ils devaient d'abord organiser leur profession, mais ils visaient aussi à la faire reconnaître dans la société, et ce, aux premières places de l'échelle sociale. Ce dernier objectif appelait une accréditation à la fois professionnelle (ou technique), culturelle et sociale. En ce sens, notre recherche s'apparente à celles de Jacques

(1882-1947)», RHAF, 48,4 (printemps 1995): 483-508. La notion d'élite doit être prise ici au sens très large d'un groupe d'individus qui occupent le sommet d'une hiérarchie collective dans les domaines de l'économie, de la politique ou de la culture (ou selon n'importe quelle combinaison de ces trois dimensions).

5. George Weisz, «The Geographical Origins and Destinations of Medical Graduates in Quebec, 1834-1939», Histoire sociale/Social History, 19,37 (mai 1986): 93-119.

6. Jacques Bernier, La médecine au Québec. Naissance et évolution d'une profession (Québec, Les Presses de l'Université Laval, 1989), 207 p.; Denis Goulet et André Paradis, Trois siècles d'histoire médicale au Québec. Chronologie des institutions et des pratiques médicales (1639-1939) (Montréal, VLB éditeur, coll. «Études québécoises», 1992), 527 p.

7. Fernand Ouellet, Le Bas-Canada 1791-1840. Changements structuraux et crise (Ottawa, Éditions de l'Université d'Ottawa, 1976), 541 p., chapitre VII.

8. Dans cet esprit, signalons deux numéros spéciaux de la revue Recherches sociographiques, l'un $(16,1,1975)$ consacré à la sociologie de la santé, l'autre $(19,2,1978)$, aux professions en général. Voir aussi Jacques Brazeau, «The Practice of Medicine in Montreal», Bernard Blishen, Frank E. Jones, John Porter, Kaspard Maegele, eds., Canadian Society (Toronto, MacMillan of Canada, 1961), 233-248; Pierre Saint-Arnaud, La société québécoise et sa pratique médicale, mémoire de maîtrise (sociologie), Université Laval, 1967, 154 p. Chez les historiens, mentionnons encore la thèse récente de Renald Lessard, Pratique et praticiens en contexte colonial: le corps médical canadien aux $17 e$ - 18 e siècles, thèse de doctorat (histoire), Université Laval, 1994, 795 p., et l'importante monographie que Denis Goulet a consacrée à l'histoire de la Faculté de médecine de l'Université de Montréal, Histoire de la faculté de médecine de l'Université de Montréal, 1843-1993 (Montréal, VLB éditeur, coll. "Études québécoises», 1993), 502 p. 
Bernier et Claudine Pierre-Deschêne pour le Québec, de Robert Douglas Gidney et W. P. J. Millar pour l'Ontario, d'Olivier Faure et Jacques Léonard pour la France, de Noël Parry, José Parry et M. Jeanne Peterson pour l'Angleterre ${ }^{9}$. En accord avec les directions d'analyse ouvertes par le sociologue Eliot Freidson aux États-Unis ${ }^{10}$, il s'agit de s'interroger sur les facteurs internes (stratégies d'autopromotion) et externes (réception dans la population) d'ascension vers le monde des élites et de la notabilité, ce concept faisant surtout référence aux normes de la bienséance et de la rectitude, de l'estime et de la respectabilité collective. C'est annoncer en même temps notre thèse: la position sociale du médecin, dans ce genre de société, devait moins à ses assises économiques (que nous nous garderons toutefois de sous-estimer) qu'à l'autorité et au rang qui lui étaient reconnus dans l'ordre du prestige et de la moralité publique. Par ailleurs, nous soutiendrons que son autorité et son rang étaient dus à son savoir, bien sûr, mais plus encore au statut qu'il était parvenu à acquérir en dehors de sa pratique professionnelle.

Sur un autre plan, il nous faudra aussi commenter ce type de collectivité qui permettait à certains acteurs de s'élever dans la hiérarchie en suivant des voies et des stratégies principalement socioculturelles. Enfin, le recours au concept d'élite révèle un autre choix théorique. Nous supposons que la structure du pouvoir dans la société saguenayenne était fragmentée et que cette pluralité donnait lieu à diverses aires et positions d'influence. En ce sens, notre analyse s'inscrit dans la tradition de Pareto (Traité de sociologie générale, 1916), Kolabinska (La circulation des élites en France, 1912), Raymond Aron, Robert A. Dahl et Nelson W. Polsby ${ }^{11}$. Mais à

9. Jacques Bernier, La médecine au Québec..., 207; Claudine Pierre-Deschênes, «Santé publique et organisation de la profession médicale au Québec, 1870-1918», RHAF, 35,3 (décembre 1981): 355-375; Robert-Douglas Gidney, W. P. J. Millar, Professional Gentlemen: the Professions in Nineteenth-Century Ontario (Toronto, University of Toronto Press, 1994), 505 p., chapitre 5; Olivier Faure, «Lyons Doctors in the Nineteenth Century: an Exceptional Social Union", Journal of Social History, 10,4 (juin 1977): 508-523; Jacques Léonard, Les médecins de l'Ouest au XIXe siècle (Paris, diffusion H. Champion, 1978), 3 vol., 1570 p.; Noël Parry, José Parry, The Rise of the Medical Profession (Londres, Croom Helm, 1976), 282 p.; M. Jeanne Peterson, The Medical Profession in Mid-Victorian London (Berkeley/Los Angeles, University of California Press, 1978), $406 \mathrm{p}$.

10. Eliot Freidson, Profession of Medicine: a Study of the Sociology of Applied Knowledge (New York, Harper \& Row Publishers, 1970), 409 p.

11. Raymond Aron, «Note sur la stratification du pouvoir», Revue française de science politique, 4,1 (1954): 469-483; Raymond Aron, «Classe sociale, classe politique, classe dirigeante», Archives européennes de sociologie/European Journal of Sociology, 1,2 (1960): 260-282; Robert A. Dahl, «The Concept of Power», Behavioral Science, 2 (1957): 201-215; «A Critique of the Ruling Elite Model», American Political Science Review, 52 (1958): 463-469; Nelson W. Polsby, Community Power and Political Theory (New Haven [CN], Yale University Press, 1963), 144 p. 
l'échelle suprarégionale, le modèle des classes sociales retrouve tous ses droits, la perspective macro-sociale faisant apparaître des rapports sociaux fondamentaux sur lesquels les différentes «élites» peuvent être réalignées. On rejoint ici l'autre grande tradition sociologique, où se côtoient Gaetano Mosca ${ }^{12}$ et Marx, selon laquelle les élites se fondent dans une même classe dirigeante.

Un mot sur les sources et les données utilisées. Ce sont d'abord des données orales provenant de deux corpus d'entrevues. Le premier résulte de 150 entretiens réalisés par l'auteur entre 1968 et 1985 auprès de personnes âgées de plus de 75 ans, principalement des agriculteurs; une dizaine de ces informateurs étaient des médecins ${ }^{13}$. Le second corpus comprend plus de 900 entrevues conservées aux Archives nationales du Québec à Chicoutimi (Mémoires d'Anciens, ANQC) et réalisées entre 1930 et 1980 par les soins de la Société historique du Saguenay (également auprès d'informateurs âgés pour la plupart de 75 ans et plus). À propos de ce corpus, soulignons que les entrevues les plus anciennes témoignent des premières décennies du peuplement saguenayen. Les quotidiens régionaux publiés entre 1890 et 1940 - principalement le Progrès du Saguenay - ont aussi été mis à profit, de même que la correspondance des évêques du diocèse de Chicoutimi. Le fichier de population BALSAC ${ }^{14}$ et les recensements du gouvernement canadien ont été utilisés pour dresser le profil démographique des médecins. Enfin, nous avons exploité à fond les procès-verbaux des réunions de la Société médicale des médecins des districts de Chicoutimi, Saguenay et Lac-Saint-Jean, fondée à Chicoutimi en $1900^{15}$.

12. Gaetano Mosca, The Ruling Class (New York, McGraw-Hill, 1939), 514 p.

13. Une grille-questionnaire spécifique a été élaborée pour ces dernières entrevues (voir Document II-C-240 de l'IREP).

14. Ce fichier contient toutes les familles reconstituées de la région du Saguenay $(\mathrm{N}=125000)$ pour la période 1842-1971. Voir Gérard Bouchard, Raymond Roy, Bernard Casgrain, Reconstitution automatique des familles. Le système SOREP (Chicoutimi, Université du Québec à Chicoutimi, 1985), 2 vol., 745 p., Dossier n 2; Gérard Bouchard, Raymond Roy, Bernard Casgrain, Michel Hubert, «Fichier de population et structures de gestion de base de données: le fichier-réseau BALSAC et le système INGRES/INGRID», Histoire \& Mesure, 4,12 (1989): 39-57.

15. Normand Perron, Un siècle de vie hospitalière au Québec. Les Augustines et l'HôtelDieu de Chicoutimi, 1884-1984 (Sillery/Chicoutimi, Presses de l'Université du Québec/Les Augustines de la Miséricorde de Jésus, 1984), 439 p., a déjà fait une heureuse utilisation de cette source que nous avons dépouillée en 1972-1974 aux Archives du Petit séminaire de Chicoutimi. Il s'agit d'une version microfilmée de deux volumes manuscrits (cote C-25-204a). L'original, que Perron a consulté, se trouverait aux Archives des Augustines de la Miséricorde de Jésus, de Chicoutimi. 


\section{2 - PROFIL DÉMOGRAPHIQUE}

Il n'y eut pas de médecin résident au Saguenay avant 1846, année où P.-C.-A. Dubois vint s'établir à Chicoutimi (voir carte). Il quitta la région en 1866, où il avait été rejoint entre-temps par Vincent Madoc Martin qui y pratiqua jusqu'à sa mort en 1874. D'autres suivirent bientôt: Cimon, Beauchamp, Blair, Caron, Riverin, etc. En 1871, on comptait 4 médecins dans l'ensemble de la région (soit un pour 4373 habitants), 6 en 1881 (ratio: 1/4 158) et 10 en 1901 (1/3 737). Durant cette période, l'indice pour la province de Québec passait de 1/1305 à $1 / 733$. À l'échelle des comtés, le Saguenay se classait parmi les régions les plus mal desservies ${ }^{16}$. Pour le $\mathrm{XX}^{\mathrm{e}}$ siècle, aucune source ne fournit de données agrégées sur les effectifs. Les procès-verbaux de la Société médicale permettent de combler en partie cette lacune. On relève que le ratio s'améliore progressivement, s'établissant à $1 / 2324$ en 1940, ce qui était encore loin toutefois de l'indice québécois au même moment (1/1054). De plus, ces mesures sont un peu trompeuses dans la mesure où les effectifs médicaux étaient inégalement répartis sur le territoire saguenayen, les noyaux urbains étant beaucoup mieux desservis que les campagnes. Ainsi, fondée en 1854, la paroisse de Roberval ne put attirer son premier médecin qu'en $1879^{17}$. À l'est du lac Saint-Jean, Alma ne fut desservie qu'en 1893, soit près d'une trentaine d'années après le début du peuplement. Au début des années 1890, plusieurs paroisses du lac Saint-Jean (Chambord, Lac Bouchette, Saint-Gédéon, Saint-Jérôme...) essayaient en vain de recruter auprès du corps médical ${ }^{18}$. Une compilation effectuée à l'aide du fichier BALSAC montre que la proportion des paroisses rurales desservies par un médecin résident a oscillé entre $1 / 15$ et $1 / 4$ avant 1950. À l'opposé, presque toutes les localités urbaines comptaient au moins un médecin.

Les membres du corps médical étaient relativement sédentaires, environ $70 \%$ des médecins n'ayant jamais migré à l'intérieur du Saguenay, une fois établis. Par contre, bon nombre d'entre eux $(40 \%$

16. Voir Jacques Bernier, «Les praticiens de la santé au Québec, 1871-1921. Quelques données statistiques», Recherches sociographiques, 20,1 (janvier-avril 1979): 41-58, tableau 3. G. Weisz, «The Geographical Origins...», 93-119, fournit des chiffres un peu différents pour le Québec, mais l'écart avec le Saguenay et la tendance générale restent similaires (voir aussi: George Weisz, «Origines géographiques et lieux de pratique des diplômés en médecine au Québec de 1834 à 1939», Marcel Fournier, Yves Gingras, Othmar Keel, dir., Sciences \& Médecine au Québec: perspectives sociohistoriques (Québec, Institut québécois de recherche sur la culture, 1987), 129-170.

17. Elle le dut à une formule coopérative astucieuse qui avait déjà été pratiquée ailleurs au Québec et en France, notamment: les familles intéressées souscrivaient à une sorte d'abonnement annuel de $4 \$$ (Rossel Vien, Histoire de Roberval, cœur du Lac-Saint-Jean, 1855-1955 (Roberval, Éditions du Centenaire, coll. «Société historique du Saguenay», $\left.\mathrm{n}^{\circ} 15,1955\right), 97$.

18. Le Progrès du Saguenay, 24 avril 1890, 2; 26 novembre 1891, 2. 


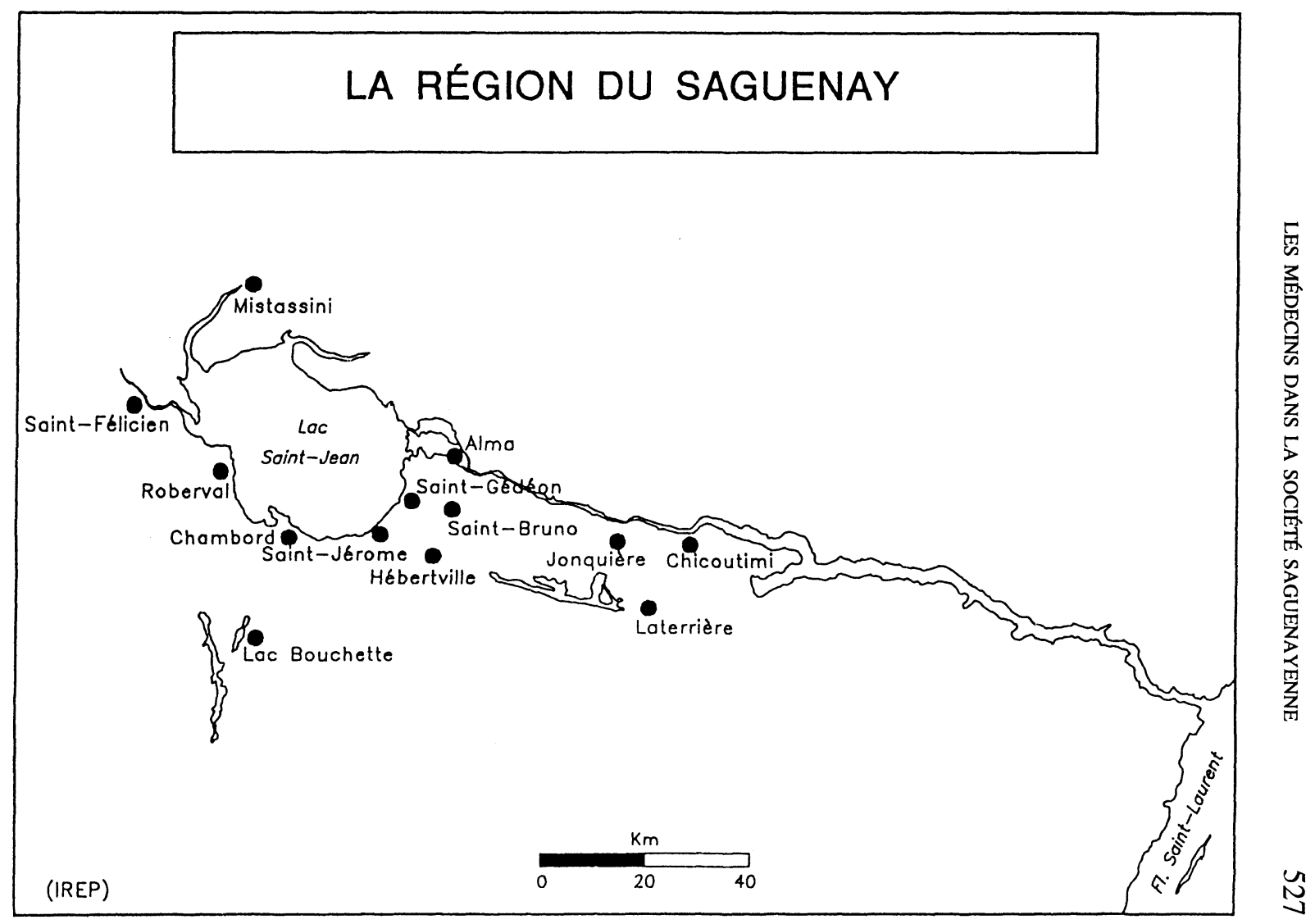


au moins) ont quitté la région avant leur retraite. En ce qui concerne la fécondité, leur comportement se distinguait peu de l'ensemble de la population. Les médecins mariés avant 1911 ont eu 10 enfants en moyenne (naissances vivantes). Ils semblent s'être adonnés à la contraception à partir de la décennie 1912-1921, soit un peu plus tôt que les autres groupes sociaux. Enfin, on ne recense aucun médecin de sexe féminin au cours de la période étudiée. On sait que c'est en 1924 seulement que la première femme fut admise dans une université francophone québécoise, en l'occurrence l'Université de Montréal ${ }^{19}$.

\section{3 - LA LUTTE POUR LA RECONNAISSANCE PROFESSIONNELLE}

Ayant ainsi assuré leur relève et la croissance de leurs effectifs, les médecins se sont heurtés jusqu'à la fin de la période étudiée à un problème d'accréditation auprès de la population. La culture populaire (urbaine et rurale) disposait déjà d'un savoir spécialisé en matière de prévention et de thérapie ${ }^{20}$. Un arsenal abondant de recettes et de remèdes était déjà en usage. Les familles maîtrisaient ces connaissances et se les transmettaient d'une génération à l'autre. Au-delà de la sphère domestique, on pouvait aussi recourir aux services de divers intervenants, allant des sages-femmes et des ramancheurs aux soigneuses et prêtres-guérisseurs. Comment les médecins s'y sont-ils pris pour se tailler une place au sein de cet univers socioculturel et y intégrer un savoir qu'ils tenaient pour supérieur ${ }^{21}$ ? On sait qu'offi-

19. Même au début des années 1950 , le pourcentage général des admissions féminines dans les universités québécoises était encore presque négligeable (moins de 10\%). Cette situation tient à ce que les collèges classiques pour filles ont été créés tardivement (début du $\mathrm{XX}^{\mathrm{e}}$ siècle) et qu'ils ont eu de la difficulté à se faire reconnaître l'équivalence avec le cours classique masculin (à partir de 1938 seulement en ce qui concerne les collèges relevant de l'Université Laval). Nous devons ces informations à la courtoisie de Johanne Daigle, Jacques Bernier et Denis Goulet. Pour une perspective plus étendue sur le sujet, voir Deborah Gorham, «No Longer an Invisible Minority: Women Physicians and Medical Practice in Late Twentieth-Century North America», D. Dobb et D. Gorham, Caring and Curing (Ottawa, University of Ottawa Press, Historical Perspectives on Women and Healing in Canada, 1994), 183-211; D. Goulet, Histoire de la faculté de médecine...

20. Ce fait est bien connu. Il est particulièrement bien illustré dans les travaux de F. Saillant sur l'est du Québec. Voir Francine Saillant, «Le rhume et la grippe. Recettes québécoises de médecine populaire», Ethnologie française, 4,1 (1991): 11-30; Francine Saillant, «Savoirs et pratiques des femmes dans l'univers ethnomédical québécois», Folklore canadien/ Canadian Folklore, 14,1 (1992): 47-72, etc. En ce qui concerne le Saguenay, les corpus de données orales en livrent de riches aperçus; voir aussi Carole Asselin, Bertrand Bergeron, JeanClaude Dupont, Yves Lacasse, Corpus des faits ethnographiques québécois, Saguenay-LacSaint-Jean (Québec, Ministère du Loisir, de la Chasse et de la Pêche, 1982), 433 p.

21. Une question de ce genre est au cœur de l'enquête exemplaire conduite par Yvonne Turin, Affrontements culturels dans l'Algérie coloniale. Écoles, médecines, religions, 1830-1880 (Paris, Maspéro, 1971), 434 p., sur l'impact culturel de la médecine savante française en Algérie au XIX ${ }^{\mathrm{e}}$ siècle. Dans ce cas toutefois, l'effort d'acculturation s'est soldé par un échec, les deux «mondes» demeurant imperméables l'un à l'autre. 
ciellement les médecins dédaignaient les recettes populaires et les praticiens non diplômés. Ils leur opposaient un savoir et une technologie appuyés sur la rigueur de la science et l'autorité de l'université. Dans leur esprit, la préséance octroyée à la médecine savante allait de soi, puisque comme l'avaient démontré la vieille tradition clinique issue de l'École de Paris, puis Claude Bernard dans son Introduction à l'étude de la médecine expérimentale (1865), les règles de la méthode scientifique étaient objectives et universelles.

Il est vrai qu'entre 1847 et 1907, la formation du médecin québécois a connu une première uniformisation ${ }^{22}$ et la pratique médicale elle-même a été encadrée et professionnalisée, comme en font foi les pouvoirs accrus dévolus au Collège des médecins et chirurgiens ${ }^{23}$. Au strict plan du savoir, la médecine savante - surtout en milieu urbain - disposait de puissants atouts: à la fin du XIX $\mathrm{X}^{\mathrm{e}}$ siècle, l'homme de l'art savait ausculter le cœur et les poumons, analyser le pouls, nettoyer les plaies à l'aide de désinfectants efficaces, réprimer les hémorragies; il combattait la variole et quelques autres maladies infectieuses par la vaccination - objet d'effroi autant que d'émerveillement auprès de la population; grâce à l'asepsie et aux anesthésiques, diverses interventions chirurgicales étaient effectuées dans d'assez bonnes conditions pour en assurer la réussite; en outre, le médecin possédait des notions précises d'hygiène personnelle et de santé publique. En ce qui concerne l'outillage, il disposait de quelques instruments qui, à l'efficacité proprement dite, ajoutait l'avantage de faire impression sur les patients et leurs proches. C'est avec un sentiment mêlé de crainte et d'admiration qu'on observait le médecin ouvrir sa trousse pour en retirer un stéthoscope ou un thermomètre, une seringue, un scalpel, un forceps ${ }^{24}$. Enfin, en ce qui concerne les remèdes, outre les pilules, sirops, onguents et autres expédients de la médecine courante, le médecin pouvait compter sur quelques ressources peu accessibles à la médecine populaire, comme les analgésiques à base d'opium et de morphine ${ }^{25}$.

22. Par exemple: Charles-Marie Boissonnault, Histoire de la faculté de médecine de Laval (Québec, Les Presses universitaires Laval, 1953), 436 p.

23. Jacques Bernier, La médecine au Québec....

24. Ibid., 111-157. On peut mentionner aussi l'ophtalmoscope et le laryngoscope.

25. Il est toutefois difficile d'évaluer exactement l'efficacité des atouts scientifiques et techniques dont disposait le médecin. Les indicateurs démographiques sont ici discordants. Par exemple, durant les décennies correspondant à l'implantation des médecins dans l'ensemble de la région (soit entre 1880 et 1930), la mortalité maternelle (femmes en couches) et la mortalité périnatale (mortalité des enfants durant leur première semaine) n'ont guère bougé. Cependant, la mortalité néonatale (mortalité des enfants durant le premier mois) a décliné dès après 1900 . Ces données sont tirées de Danielle Gauvreau, «Donner la vie et en mourir: la mortalité des femmes en couches au Québec avant 1960», Dennis D. Cordell, Danielle Gauvreau, Raymond R. 
Mais cet arsenal ne suffisait pas à imposer le médecin de façon exclusive à des populations rurale et urbaine, qui le mettaient en parallèle ou en concurrence avec d'autres «spécialistes» plus familiers, comme les ramancheurs, les sages-femmes, les curés-thaumaturges, les guérisseurs. Ce dernier type regroupait ceux ou celles qui avaient reçu un don à la naissance (le septième fils, la septième fille...) ou qui l'avaient hérité d'un parent (faire passer les coliques ou le mal de dents, arrêter le sang, supprimer les varices, les verrues, etc.) et tous ceux ou celles qu'on qualifiait de charlatan ${ }^{26}$. Si les guérisseurs appartenaient ordinairement à la société locale, les charlatans étaient la plupart du temps des étrangers: colporteurs, quêteux, personnages de cirques ambulants, astrologues, voyants ou «savants des académies», que le train déposait au matin dans une gare et ramenait le lendemain. Vers la fin du XIX ${ }^{\mathrm{e}}$ siècle, plusieurs curés s'inquiétaient de leur nombre grandissant dans les campagnes. Les journaux en faisaient état, ordinairement pour mettre la population en garde. Un matin de 1889 , un certain «Dudevoir» vendait ses remèdes à la sortie de la messe devant la cathédrale de Chicoutimi; apparemment bien connu de la foule, il s'y trouvait des partisans et des détracteurs qui se manifestèrent bruyamment, ce qui aurait donné lieu à une scène jugée «disgracieuse» et nécessité l'intervention des constables ${ }^{27}$. Un dénommé Georges Tucker, qui se présentait comme un célèbre «guérisseur indien» signalé un peu partout au Québec, séjourna aussi à Chicoutimi, en même temps que le «professeur Comot» (ou «Cornot»?), annoncé, lui, comme un membre correspondant de la Faculté de médecine de Paris et ex-interne des hôpitaux militaires de Bordeaux. Ils prononçaient des conférences, recevaient en consultation... ${ }^{28}$. Un autre type d'intervenants, la soigneuse, était assurément plus inoffensive et bien connue des familles. C'était souvent une veuve, âgée de préférence, bien connue dans sa paroisse; elle dispensait des soins mineurs: pansements, emplâtres, frictions, tisanes, «sueries». Enfin, l'Indien ou l'Indienne (la «Sauvagesse»), qui connaissait les herbages et les secrets des plantes, était un personnage important que l'on consultait toutefois moins volontiers: sa condition

Gervais, Céline Le Bourdais, dirs., Population, reproduction, sociétés. Perspectives et enjeux de démographie sociale (Montréal, Les Presses de l'Université de Montréal, 1993), 235-256, et de travaux (non publiés) de Raymond Roy.

26. Le mot, qui avait une connotation péjorative, appartient surtout au vocabulaire de la répression, ce qui rend son emploi difficile dans une analyse comme celle-ci. Voir à ce propos Toby Gelfand, «Medical Professionals and Charlatans. The Comité de salubrité enquête of 179091», Histoire sociale/Social History, 11,21 (mai 1978): 62-97.

27. Le Progrès du Saguenay, 31 octobre 1889, 3.

28. Le Progrès du Saguenay, 8 juin 1893, 3. 
ethnique en faisait un étranger et l'assimilait parfois au charlatan. Ces acteurs ne se trouvaient pas tous dans un même rapport de compétition ou de rivalité avec le médecin. Des nuances s'imposent ici. La sagefemme, par exemple, remplaçait assez souvent l'homme de l'art avec son accord, sinon à sa demande quand il n'était pas disponible - dans les paroisses éloignées, mal desservies. Dans plusieurs cas, le médecin lui-même aidait à la formation de la sage-femme et en faisait une sorte d'assistante ${ }^{29}$. De même, la soigneuse bénéficiait d'une certaine tolérance comme acteure marginale, agissant plus ou moins en complémentarité avec le médecin. Mais les autres intervenants ont toujours été perçus comme des concurrents menaçants qui exerçaient illégalement la profession médicale et devaient être systématiquement dénoncés.

Très tôt, les médecins se sont inquiétés de l'action de ces différents intervenants et se sont employés à les dénoncer pour les faire interdire. Ce sujet a souvent été abordé par des historiens et des ethnologues, dans le cadre québécois comme ailleurs ${ }^{30}$. Cette offensive contre la médecine populaire a été dirigée soit contre les praticiens, soit contre la culture elle-même. Par le biais de ces démarches entreprises explicitement dans l'intérêt des patients, les médecins cherchaient, bien sûr, à éliminer les pratiques inefficaces inspirées par la superstition, et à neutraliser l'intervention d'imposteurs auprès des familles non prévenues. Cet objectif officiel et louable en dissimulait

29. À l'occasion, un homme pouvait aussi exercer le métier de «sage-femme» ou d'accoucheur. Nos sources mentionnent deux cas de ce genre, l'un à Hébertville (Lac-SaintJean), l'autre à Laterrière (au sud de Chicoutimi). Notons par ailleurs que notre analyse des rapports entre sages-femmes et médecins en milieu rural est en discordance avec celle que propose Hélène Laforce, Histoire de la sage-femme dans la région de Québec (Québec, Institut québécois de recherche sur la culture, 1985), 237 p., pour l'ensemble du Québec. Cette auteure fait du reste référence, à propos d'une paroisse rurale du Saguenay, à des poursuites intentées par le Collège des médecins contre des sages-femmes (pages 110-111, 133). Pour l'instant, les archives criminelles du Saguenay ne sont pas accessibles. Cette question reste donc en suspens. Signalons toutefois qu'en Ontario (seconde moitié du XIX ${ }^{e}$ siècle), J. T. H. Connor, «Larger Fish to Catch here than Midwives: Midwifery and the Medical Profession in Nineteenth-Century Ontario", Dianne Dodd and Deborah Gorham, eds., Caring and Curing (Ottawa, University of Ottawa Press, 1994), 103-134, chapitre 5, a démontré que plusieurs médecins appuyaient le travail des sages-femmes. À supposer que notre interprétation soit la bonne, le Saguenay ne serait donc pas un cas isolé.

30. En plus des ouvrages déjà mentionnés (par exemple: J. Bernier, Les médecins au Québec..., 95-101), voir Gilles Brunel, «La culture populaire en procès permanent: le cas des guérisseurs traditionnels au Québec», Sociologie et Sociétés, 11,1 (avril 1979): 147-165; Matthew Ramsey, «Medical Power and Popular Medicine: Illegal Healers in Nineteenth-Century France», Journal of Social History, 10,4 (juin 1977): 560-587. Mentionnons aussi les travaux en cours de Francine Saillant sur le savoir médical populaire au Québec et les efforts déployés par la médecine savante pour le réprimer ou le convertir (on peut suivre la marche de cette recherche dans les rapports annuels de l'IREP). 
un autre: acquérir le monopole des soins de santé à des fins de promotion économique et sociale. L'action du corps médical fut d'abord dirigée contre ce qu'on appelait les «illégaux», c'est-à-dire ceux qui dispensaient des soins sans diplôme ou sans licence. Elle visait ensuite ceux qu'on appelait les charlatans, la plupart du temps des noninstruits qui faisaient le métier de guérir grâce à des recettes miracles, à la connaissance de secrets ou à l'invocation de puissances occultes. $\mathrm{Au}$ cours du dernier tiers du XIX ${ }^{\mathrm{e}}$ siècle, la législation québécoise accrut les pouvoirs du Collège des médecins et chirurgiens qui entreprit de poursuivre lui-même les contrevenants devant les tribunaux. Le Collège publiait un registre officiel des médecins qui étaient en règle et, au cours des années 1880 , il mandata un médecin pour agir en son nom dans chaque district judiciaire du Québec. Au Saguenay, l'action du Collège était soutenue par la Société médicale des médecins des districts de Chicoutimi, Saguenay et Lac-Saint-Jean, créée à l'automne 1900. Cet organisme, qui ne fut jamais constitué en corporation, reprenait le modèle de la Société médicale de Québec et de plusieurs autres, réunies en fédération en $1922^{31}$. L'assemblée de fondation, qui a eu lieu à Chicoutimi le 11 novembre 1900 , réunit 10 médecins et officialisa les objectifs énoncés lors d'une réunion préparatoire tenue le mois précédent (15 octobre):

- Rehausser le niveau de la science médicale en assurant une meilleure communication des connaissances scientifiques que chacun aura acquises par la lecture des «journaux» et par son expérience personnelle.

- Employer tous les moyens pour lutter contre le charlatanisme; dénoncer la pratique illégale de la médecine.

- Hausser et uniformiser les tarifs.

Les 110 réunions («conventions») tenues par la Société entre 1900 et 1940 furent presque exclusivement consacrées à ces trois sujets, les deux derniers l'emportant toutefois sur le premier. Dès leur première réunion, les médecins déplorèrent, en effet, le grand nombre de charlatans et de médecins non licenciés (par le Collège des médecins et chirurgiens) ${ }^{32}$. Ils soulignaient aussi l'inefficacité de la loi qui, au surplus, était appliquée trop mollement à leur goût. Ce point est à retenir, car, périodiquement, les procès-verbaux des réunions laissent

31. Voir Albert Jobin, «Les débuts de la Société médicale de Québec», Laval Médical, 7,7 (septembre 1942): 333-340; C.-A. Gauthier, «Histoire de la Société médicale de Québec», Laval Médical, 8,1 (janvier 1943): 62-121; D. Goulet, A. Paradis, Trois siècles d'histoire médicale au Québec..., section III.

32. L'accréditation par le Collège équivalait à un certificat d'habilitation; elle comportait aussi une assermentation de l'intéressé. 
à entendre que le législateur et la magistrature n'étaient pas très enclins à réprimer le personnel médical populaire, lequel aurait donc trouvé une certaine sympathie et ce, même parmi les notables. À diverses reprises au cours de la période considérée ici, la Société mit sur pied ou relança un comité chargé de relever les cas de pratique illégale et de faire traduire les contrevenants en justice. Plusieurs cas de poursuite ont effectivement été rapportés, le plus souvent contre de présumés médecins qui n'étaient pas en règle avec le Collège, ou encore contre des charlatans et des rebouteux qui étaient mis à l'amende ou, plus rarement, emprisonnés ${ }^{33}$. Dans d'autres cas, beaucoup plus délicats, le mécontentement des médecins était dirigé contre des gens d'Église: des curés qui auraient administré des soins médicaux aux malades, des religieuses hospitalières (de Chicoutimi et de Roberval) qui auraient prescrit des médicaments et appliqué certains traitements aux patients. Dans ces cas-là, on envoyait quelques médecins en délégation auprès de l'évêque, comme il arriva, par exemple, en 1934 et en 1941.

La vigilance du corps médical ne s'arrêtait pas là; elle s'étendait aussi à la production et à la vente des médicaments. En vertu de la loi canadienne de 1874 (entrée en vigueur en 1875), il était relativement facile de fabriquer et de vendre des médicaments, et ce, sans même en indiquer la composition sur le contenant. À partir de 1908, une nouvelle loi imposa des restrictions quant à l'usage des «médecines patentées» (remèdes, médicaments brevetés), tout en octroyant un certain pouvoir de contrôle aux médecins et pharmaciens dûment autorisés ${ }^{34}$. Avant 1908, les médecins dénonçaient les vendeurs ambulants de remèdes parce qu'ils ne payaient pas de taxes municipales, ce qui donnait lieu à une concurrence jugée injuste pour les praticiens résidents. Néanmoins, il semble que la loi n'apporta pas la solution désirée tant les transgressions furent nombreuses, d'où sans doute les amendements qui lui furent apportés, notamment en 1919 et en 1925. À plusieurs reprises au cours de ses réunions, la Société renouvela ses plaintes contre les colporteurs et même contre des épiciers qui se livraient au commerce illicite de remèdes ${ }^{35}$. Pendant toute cette

33. Il nous a été impossible d'analyser ces dossiers judiciaires, les archives criminelles déposées aux Archives nationales du Québec à Chicoutimi étant en cours de classement.

34. «Loi concernant les médicaments dits proprietary ou les médicaments brevetés», sanctionnée le 20 juillet 1908. Sur la loi de 1874, voir, parmi d'autres, Johanne Collin, Denis Béliveau, Histoire de la pharmacie au Québec (Montréal, Musée de la pharmacie du Québec, 1994), 333 p., chapitre 5.

35. En fait, tous les marchands généraux étaient un peu apothicaires; on pouvait se procurer auprès d'eux les matériaux de base de la pharmacie familiale: graine de lin, huile de castor, gingembre, liniment, et le reste. Quant aux colporteurs, il en est fait état couramment 
période, les griefs étaient transmis au Collège des médecins et chirurgiens qui intentaient les procédures. La Société médicale se plaignait de l'inefficacité de ce système; elle aurait souhaité entreprendre ellemême, et plus vigoureusement, les poursuites.

L'insertion des médecins dans cette société était ralentie sur un autre front: la population elle-même demeurait largement fidèle à ses auxiliaires traditionnels et manifestait souvent des réticences à l'endroit de ce nouvel acteur que constituait le diplômé de la Faculté. Le ramancheur, la sage-femme, le guérisseur étaient des figures très familières et très appréciées, envers lesquelles la population éprouvait une sorte de complicité, une solidarité, en particulier envers le ramancheur, dont le savoir se transmettait de père en fils ${ }^{36}$. Il parlait la langue du peuple auquel il appartenait, et disait devoir sa compétence à une assistance constante de Dieu. Du reste, tout comme la sagefemme et le guérisseur, il était ordinairement de mœurs irréprochables. «Ti-Zèbe» Simard, de Saint-Félicien (Lac-Saint-Jean), était de ceuxlà. Il avait su se mériter la plus grande estime de ses concitoyens qui le consultaient sur diverses affaires et qui en avaient fait un peu leur médecin. Au début clu siècle, un employé de la scierie de Roberval s'était grièvement blessé à une jambe et les deux médecins accourus auprès de lui s'étaient prononcés pour l'amputation. Mais l'homme s'y opposa et demanda plutôt l'intervention de Ti-Zèbe qui, finalement, lui aurait sauvé la jambe. L'événement donna naissance à une légende selon laquelle l'un des deux médecins aurait offert $3000 \$$ à Ti-Zèbe pour apprendre son secret, mais en vain... ${ }^{37}$.

Les témoignages abondent pour illustrer les réticences et même le refus dont le médecin était l'objet. D'abord, il faut rappeler que, d'une manière générale, une relation assez robuste au corps caractérisait ces classes populaires (urbaine et rurale). On se soignait relativement peu et, autant que possible, on le faisait soi-même en recourant aux ressources domestiques. La mère jouait ici un rôle important; c'est ordinairement elle qui appliquait les onguents, frictions, tisanes et sirops à base de gomme d'épinette, de miel, de poivre, de graine de lin, d'huile de castor, d'alcool etc. On voyait aussi, dans des paroisses

\footnotetext{
jusque dans les années 1930, surtout dans les campagnes: des «Syriens» vendeurs d'herbages; des rapatriés des États-Unis qui offraient les Rival Herb Tablets contre les «maladies de femmes», le sang impur et le mal de tête; des gens des "vieilles paroisses» (Charlevoix, Côtede-Beaupré, Bas-Saint-Laurent) qui vendaient des «remèdes de Sauvages»... À ce propos, on consultera J. Collin, D. Béliveau, Histoire de la pharmacie au Québec...

36. C'était le cas notamment des Boily, originaires de Charlevoix, dont la lignée s'est perpétuée sur quatre ou cinq générations au Saguenay.

37. ANQC, Mémoire $\mathbf{n}^{\circ} 542,5-6$.
} 
particulièrement isolées, des adultes réduire eux-mêmes des fractures mineures qu'ils s'étaient infligées, nettoyer leurs plaies à vif au moyen d'un couteau, pratiquer des incisions pour crever un abcès ou soulager une enflure... Selon plusieurs témoignages, on soignait assez couramment les plaies en les léchant pour y déposer de la salive. À l'occasion, on pratiquait aussi la saignée au moyen de lancettes improvisées. C'est ce que fit Prudent Marceau de Mistassini, lorsqu'il apprit du médecin que sa femme était «décomptée»; il entreprit lui-même de la saigner, ce qui aurait permis de prolonger sa vie de quelques années $^{38}$. Tel autre, qui s'était blessé au pied avec sa hache, arrêta l'hémorragie en appliquant sur la plaie de la mousse (spore) de vessede-loup desséchée mêlée d'excréments de cheval ${ }^{39}$, etc. Selon leurs dires, les gens de condition modeste éprouvaient généralement de la gêne à faire venir un médecin. De préférence, on sollicitait d'abord un ramancheur, un guérisseur ou une soigneuse. L'intervention du médecin était associée à des circonstances très graves; on s'y résolvait quand les autres recours avaient été épuisés. Jusque dans les années 1930, dans la grande majorité des cas, l'accouchement ne faisait pas partie de ces circonstances; il se déroulait sous la direction de la sagefemme (il est vrai par ailleurs que pour les gens du peuple l'accouchement n'était pas associé à la maladie).

Lorsqu'il était appelé au chevet d'un malade, le médecin devait faire face à des attitudes réfractaires: les patients ne se montraient pas toujours coopératifs, oubliaient parfois de régler leurs comptes (problème qui refait surface aux réunions de la Société médicale), ne prenaient pas leurs remèdes tel que prescrit, ne suivaient pas les conseils... Plusieurs familles étaient un peu sceptiques et se trouvaient mal à l'aise devant une médecine qui aliénait le malade et la maladie, au sens propre comme au figuré: l'outillage et le vocabulaire de la médecine savante ne leur étaient pas familiers; on savait peu de choses sur la nature de certains médicaments prescrits; le médecin appartenait à l'univers scientifique et lointain des «grandes écoles» et des métropoles; et parfois son intervention entraînait l'hospitalisation, c'est-àdire l'éloignement du patient qui se voyait dès lors privé de l'appui des siens ${ }^{40}$. Était également associé au personnage du médecin tout ce qui concernait la vaccination, source traditionnelle de peur, d'oppo-

38. ANQC, Mémoire $n^{\circ} 233,104$. Sur le même sujet, voir aussi Mémoire $n^{\circ} 131,153$.

39. ANQC, Mémoire $n^{\circ} 61,90$. L'utilisation du fumier, notamment de la bouse de vache, dans les traitements ou remèdes populaires est assez fréquemment signalée ailleurs au Québec et en France (communications personnelles de Francine Saillant et de Françoise Loux).

40. Le premier hôpital régional fut fondé à Chicoutimi en 1884. Le deuxième fut celui de Roberval en 1918 (N. Perron, Un siècle de vie hospitalière au Québec...). 
sition et même de rébellion. Bien sûr, on pense ici aux émeutes survenues à Montréal à l'occasion de la grande épidémie de variole de $1885-1886^{41}$. Au Saguenay, l'introduction du vaccin, pour les animaux comme pour les humains, a aussi suscité bien des éotions. Encore une fois, les données orales livrent de nombreuses anecdotes à ce sujet: histoires de vaccins ruineux et inutiles, administrés par des incompétents, qui ont aggravé l'état du patient, entraîné la mort ${ }^{42}$. L'épisode le plus marquant s'est produit en 1902 à Lac-Bouchette, petite paroisse située au sud de Roberval (Lac-Saint-Jean). De nombreux cas de varicelle y furent signalés durant l'hiver parce qu'on avait trop tardé à appliquer un règlement provincial imposant la vaccination dans ce genre de situation. La maladie s'était déclarée peu avant les élections à la mairie, ce qui avait fait de la vaccination le principal enjeu électoral. Deux partis s'étaient formés, les vaccinateurs et les anti-vaccinateurs. Ces derniers l'emportèrent mais durent finalement se plier au règlement ${ }^{43}$. Ce cas n'est pas vraiment isolé; dans une circonstance analogue, en 1910, le conseil municipal de la paroisse de Saint-Bruno (Lac-Saint-Jean) dut imposer des amendes à des individus qui s'opposaient à la vaccination ${ }^{44}$. De même, dans les années 1930, des parents faisaient obstacle au travail de la première unité sanitaire de Chicoutimi en refusant de faire vacciner leurs enfants contre les maladies contagieuses les plus courantes ${ }^{45}$.

D'autres facteurs jouaient contre le corps médical. En ce qui concerne les médicaments, il subissait la concurrence des annonces publicitaires qui paraissaient régulièrement dans les hebdomadaires

41. Terry Copp, Classe ouvrière et pauvreté. Les conditions de vie des travailleurs montréalais, 1897-1929 (Montréal, Boréal Express, 1978), 213 p., chapitre 6; Michael Farley, Peter Keating, Othmar Keel, «La vaccination à Montréal dans la seconde moitié du $19^{\mathrm{e}}$ siècle: pratiques, obstacles et résistances», Marcel Fournier, Yves Gingras, Othmar Keel, dirs., Sciences \& Médecine au Québec: perspectives sociohistoriques (Québec, Institut québécois de recherche sur la culture, 1987), 87-127; Martin Tétreault, L'état de santé des Montréalais, 1880-1914 (Montréal, Université de Montréal, Université du Québec à Montréal, coll. RCHTQ, «Études et documents», $\mathrm{n}^{\circ}$ 5, 1991), 225 p. Michael Bliss, Montréal au temps du grand fléau: l'histoire de l'épidémie de 1885 (Montréal, Éditions Libre Expression, 1993), 348 p.

42. Nous avons recueilli quelques versions d'une anecdote (authentique?) mettant en vedette un jeune vétérinaire du gouvernement venu au Saguenay pour vacciner les bovins dans les années 1920. S'étant présenté chez un cultivateur, il produisit ses «pâpiers» l'autorisant à procéder. Il fut dès lors conduit dans un enclos où un énorme «beû» avait été mis à paître. Le vétérinaire s'approcha de la bête et fut aussitôt pris en chasse. Pris de panique, il pria à grands cris le cultivateur de venir le secourir. Il se serait fait répondre: «Montre-lui donc tes 'pâpiers'».

43. Le Progrès du Saguenay, 6 mars 1902, 1.

44. Jocelyn Côté, Histoire de Saint-Bruno, Lac-Saint-Jean: des origines à nos jours (Saint-Bruno, Corporation du centenaire de Saint-Bruno, 1885-1985, 1985), 96.

45. Thérèse-S. Gauthier, «Les infirmières au Saguenay-Lac-Saint-Jean», Saguenayensia, 23,1 (janvier-mars 198॥): 6-9. 
régionaux et qui étaient parfois dirigées explicitement contre les «remèdes de médecins $s^{46}$ ». Il arrivait que ces publicités accolent au produit une référence religieuse quelconque: le biphosphate de chaux des frères Maristes, la pierre miraculeuse dite «Pierre du Christ», les fameux vins Saint-Léon et Saint-Michel et celui, encore plus fameux, des pères Trappistes, les cures miraculeuses, les petits «démons» qui causent les rhumatismes ${ }^{47}$. Sur le plan de la pratique professionnelle elle-même, le médecin se trouvait en outre désavantagé du fait qu'il exigeait des honoraires beaucoup plus élevés que ceux de ses concurrents (infra). En fait, la grande majorité des sages-femmes et des soigneuses, et même plusieurs guérisseurs, opéraient gratuitement. C'était aussi le cas du prêtre, bien sûr, lorsqu'on le priait d'intervenir comme thaumaturge ${ }^{48}$. Une autre difficulté tenait au fait que plusieurs paroisses n'avaient pas de médecin; il fallait alors en faire venir un de loin et, dans les cas graves, il arrivait généralement trop tard pour faire quoi que ce soit. Ajoutons à cela qu'on ne faisait appel à ses services qu'en dernier recours et dans les cas désespérés; il lui était donc plus difficile de faire valoir sa compétence. Celle-ci était du reste régulièrement mise en doute, pour cette raison précisément. Selon un dicton peu bienveillant, si on voulait que le malade meure, il suffisait d'appeler le médecin ${ }^{49}$. Enfin, ce dernier avait la réputation d'être un peu buveur, comme l'attestent divers témoignages: des médecins qui pratiquaient «en boisson» ou qui furent soignés pour alcoolisme ou qui se livraient à la vente illégale d'alcool durant la période de prohibition (laquelle a couvert les premières décennies du $\mathrm{XX}^{\mathrm{c}}$ siècle). Certains profitaient de ce qu'ils tenaient pharmacie pour s'adonner à des prescriptions complaisantes ${ }^{50}$. D'autres, selon nos données orales, déjouaient la loi en ajoutant un peu de gomme de sapin dans le gin, sous prétexte de lui donner une propriété purgative... Les acheteurs se plaignaient un peu du mauvais goût mais s'en accommodaient

46. Voir par exemple Le Progrès du Saguenay, 6 avril 1893, 3. Il s'agit d'un remède contre le rhumatisme dont l' «action sur le système est remarquable et mystérieuse». On vante aussi un médicament qui guérit la gale sur les hommes et sur les animaux en trente minutes.

47. La prolifération publicitaire à laquelle on assiste au début du $\mathrm{XX}^{\mathrm{e}}$ siècle au Québec a été analysée par Denis Goulet, Le commerce des maladies. La publicité des remèdes au début du siècle (Québec, Institut québécois de recherche sur la culture, coll. «Edmond-de-Nevers», $\left.\mathrm{n}^{\circ} 6,1987\right), 139 \mathrm{p}$. Voir aussi Normand Perron, «Santé, maladie et publicité au Saguenay, 19001910», Saguenayensia, 26,3 (juillet-septembre 1984): 102-109.

48. Nous donnons à ce mot une acception très large, en y incluant toutes les formes d'intercession auprès de Dieu ou d'un(e) saint(e).

49. Ce qui valait aussi pour le vétérinaire, pour la même raison.

50. On sait que plusieurs médicaments avaient une teneur en alcool de $30 \%$ à $40 \%$. D'autres contenaient des substances hallucinogènes (D. Goulet, Le commerce des maladies..., chapitre 1). 
volontiers. Ces pratiques ont du reste donné lieu à des poursuites et à des condamnations ${ }^{51}$.

En conséquence, pour gagner la confiance et l'appui des familles, le corps médical a été amené à accepter certains compromis et à mettre au point une stratégie très explicite. Ainsi, dans un esprit de syncrétisme, les médecins intégrèrent à leur pratique d'importants éléments de la médecine populaire. Il pouvait s'agir de traitements ou, plus couramment, de médicaments. On trouve ainsi des médecins qui fabriquaient des sirops à base de gomme d'épinette ou qui ajoutaient aux médicaments patentés des ingrédients familiers, empruntés à la pharmacopée populaire (huile de castor, camphre, miel, graine de lin...). Les «ponces» (grogs) à base de brandy, de whisky ou de rhum étaient aussi très recommandées. On rapporte qu'un certain docteur Talbot du Lac-Saint-Jean avait mis au point un médicament contre l'épilepsie ${ }^{52}$. Un autre témoin raconte que, souffrant d'hypertension, on lui avait prescrit un traitement basé sur l'alternance de deux régimes, de quinze jours en quinze jours: l'un à base de pilules vendues par le médecin, l'autre à base de tisanes qu'il préparait lui-même avec des feuilles d'aulne ${ }^{53}$. À l'extérieur du Saguenay, on connaît aussi quelques exemples de médecins (québécois) qui ont eux-mêmes rédigé des livres de recettes dites populaires, comme l'a montré Francine Saillant dans ses travaux. Des faits semblables ont été rapportés pour d'autres régions du Québec ${ }^{54}$ et pour d'autres pays comme les États-Unis ${ }^{55}$ et la France ${ }^{56}$. Les références religieuses constituaient une autre forme de concession à la culture populaire. Après avoir administré ses traitements, le médecin rappelait fréquemment au patient l'utilité de la prière, situant ainsi son action en complémentarité avec la volonté divine, sinon dans son prolongement direct. Sur ce terrain, l'habitant se retrouvait définitivement chez lui. Dans le même esprit et en certaines occasions, le médecin pouvait aussi se

51. Voir par exemple Le Progrès du Saguenay, 17 janvier 1918, 1. Sur ce même sujet, une lettre conservée aux Archives de l'évêché de Chicoutimi (Registre des lettres, Vol. II, lettre du 25 août 1902) donne à penser que ce trafic aurait peut-être bénéficié de la solidarité d'une partie du corps médical, sinon des autorités municipales elles-mêmes.

52. ANQC, Mémoire $n^{\circ} 295,1$.

53. Corpus d'entrevues réalisées par l'auteur.

54. Voir par exemple Edmond Grignon, En guettant les ours. Mémoires joyeux d'un médecin des Laurentides (Montréal, Librairie Beauchemin Limitée, 1930), 259 p.; Claire Gervais-Roy, «Un médecin de campagne d'autrefois», Revue d'ethnologie du Québec, 3,7 (1978): 87-102.

55. Lamar Riley Murphy, Enter the Physician: the Transformation of Domestic Medicine, 1760-1860 (Tuscaloosa, University of Alabama Press, History of American Science and Technology Series, 1991), $312 \mathrm{p}$.

56. Arlette Farge, «Le médecin et l'imaginaire féminin au moment de la naissance», Revue de médecine psychosomatique et de psychologie médicale, 18,1 (1976): 23-29. 
montrer conciliant envers certains représentants du personnel thérapeutique populaire comme les soigneuses, les sages-femmes (supra) ou les ramancheurs (auxquels certains patients étaient quelquefois référés). Ceci pour les compromis. Quant à la stratégie, elle se prolongeait dans un refus de partager avec les patients des informations sur la nature exacte de remèdes prescrits et de certains soins prodigués. À la $38^{\mathrm{e}}$ «Convention» tenue le 17 juillet 1916, l'un des médecins présents expliqua à ses confrères l'intérêt qu'ils avaient à entourer de secret, autant que possible, certains remèdes et traitements qu'ils prodiguaient: ce serait une manière efficace, assurait-il, de conserver leur clientèle en maintenant les patients sous leur dépendance. Autrement, ceux-ci apprenaient, imitaient le médecin et finissaient par s'en passer. En somme, une double stratégie commandait le médecin: d'un côté, pour amadouer les familles, il imprégnait sa pratique d'emprunts à la médecine populaire; de l'autre, pour les retenir, il leur faisait sentir le poids d'un savoir que lui seul pouvait maîtriser.

Mais à l'inverse, d'autres facteurs un peu inattendus jouaient en sa faveur. Ainsi, sur le plan économique, le personnage du médecin avait parfois beaucoup en commun avec les petites gens, du moins aux premiers temps de la colonisation. Le corps médical présentait une certaine hétérogénéité de ce point de vue. Les premiers médecins du Haut-Saguenay durent occuper divers emplois dans l'administration publique pour subvenir à leurs besoins. Au Lac-Saint-Jean, vers la fin du XIX ${ }^{\mathrm{e}}$ siècle, des médecins faisaient un peu d'agriculture et d'élevage $^{57}$. Cette réalité affleure périodiquement dans les procès-verbaux de la Société médicale. À quelques reprises, celle-ci dut venir en aide à des confrères dans le besoin ou à leur veuve. La Crise frappa durement plusieurs médecins de campagne. De temps à autre, des membres de la Société se plaignaient du coût des réunions, où la bonne chère aurait pris trop de place ${ }^{58}$. Parmi les médecins qui desservaient les paroisses pauvres ou les rangs de colonisation, plusieurs se contentaient de paiements très modestes, souvent en nature (œufs, légumes, bois de chauffage) ou acceptaient simplement ce que la famille pouvait donner. À Roberval en 1879, une formule coopérative avait été mise au point par le curé pour attirer un premier médecin. Chaque famille était invitée à souscrire à un «abonnement» de $4 \$$ par année (payé moitié en argent, moitié en effets), ce qui lui donnait droit aux services du médecin, à volontée ${ }^{59}$. Une formule semblable fut

57. ANQC, Mémoire $\mathbf{n}^{\circ} 494,135-136$.

58. En 1927, il est résolu que désormais le dîner, qui se prenait habituellement dans un hôtel, serait servi dans un restaurant, ce qui en réduirait le coût de moitié $\left(69^{\circ}\right.$ "Convention» de la Société médicale).

59. Rossel Vien, Histoire de Roberval..., 97. 
appliquée quelques années plus tard à Saint-Félicien ${ }^{60}$. Les données orales recèlent de nombreux témoignages d'admiration et de reconnaissance envers des médecins particulièrement dévoués et généreux qui ne ménageaient pas leur temps et leurs peines, vivaient de peu et ne réclamaient jamais le paiement des comptes en souffrance. Leurs concitoyens les appelaient «médecins des pauvres». Ils pouvaient faire de longues distances à pied, à bicyclette, en canot ou à cheval, hiver comme été, souvent dans la tempête, le jour ou la nuit. Les colons les trouvaient parfois immobilisés par un banc de neige dans les «côtes», l'homme appuyé contre son cheval pour se réchauffer. Il fallait traverser des lacs, des rivières dont la glace cédait par endroits, et consentir parfois à de longs déplacements vers des paroisses isolées. Dans ce cas, la famille du patient se chargeait elle-même de conduire le médecin. Dans les années 1860, des gens partaient du Lac-Saint-Jean pour venir chercher le médecin à Chicoutimi et ensuite l'y ramener ${ }^{61}$.

Mais il se trouva bientôt aussi des médecins plus opulents, qui surveillaient leurs comptes et menaient grand train. Amateurs de chevaux, de voyages, de confort et de bonne table, ce sont eux surtout qui animaient les discussions sur les tarifs aux assemblées de la Société. C'était là une pomme de discorde. Certains auraient voulu imposer une échelle de tarification jugée beaucoup trop élevée par d'autres. Ce fut le sujet principal des trois premières réunions, en 1900 et en 1901, au cours desquelles on parvint à s'entendre sur un tarif unique en ce qui concerne les accouchements (l'application de forceps commandant un surplus, de même que les accouchements durant plus de six heures), les hémorragies, les extractions de dents, les anesthésies, etc. ${ }^{62}$ Ces prix furent périodiquement indexés entre 1901 et 1940 . En 1918, par exemple, ils avaient été multipliés par un facteur allant de deux à vingt, en fonction de l'intervention pratiquée, ce qui représente des augmentations moyennes bien supérieures à la hausse du coût de la vie. Mais régulièrement aussi, cette échelle était transgressée, en dépit des interdictions formelles votées aux assemblées. De nombreux médecins de campagne persistaient dans leurs habitudes, prenant le parti des familles peu fortunées. La Société formait des comités chargés de mettre au pas les irréductibles, dont le nombre, il est vrai,

60. ANQC, Mémoire $\mathrm{n}^{\circ} 62,96$.

61. On pourrait en dire autant des infirmières des colonies, qui ont eu à œuvrer, le plus souvent, dans les conditions les plus ingrates. Johanne Daigle, Nicole Rousseau et Francine Saillant, «Des traces sur la neige... La contribution des infirmières au développement des régions isolées du Québec au XX'e siècle», Recherches féministes, 6,1 (1993): 93-103.

62. L'entente était d'une grande précision, prévoyant le coût de l'extraction d'un corps étranger dans l'œil $(0,50 \$)$, la réduction d'une luxation d'un doigt $(1,00 \$)$, l'ouverture d'un abcès (de $0,50 \$$ à $1,00 \$)$, etc. 
diminuait constamment: en 1936, il n'en restait plus que cinq qui refusaient encore de signer la formule du tarif.

Les pages qui précèdent font voir le chemin étroit que devait parcourir le médecin pour s'insérer dans cette société et s'y imposer comme rouage indispensable. Aux plans du savoir et de la compétence, il devait livrer concurrence à un personnel populaire déjà bien en place au cœur de la sociabilité locale. Il devait en outre jouer subtilement de sa science, d'une part en la maintenant hors de portée du vulgaire, d'autre part en y intégrant des éléments empiriques et familiers pour amadouer les récalcitrants. Ce faisant, il veillait à créer une dépendance parmi les familles et à hausser ses honoraires en conséquence. Ce processus ne fut toutefois pas linéaire; des dissidents, qui se firent de plus en plus rares entre 1900 et 1940, prenaient le parti des petites gens dont ils partageaient un peu l'existence précaire, surtout dans les campagnes. Parallèlement, le corps médical luttait pour établir son autorité sur la diffusion des médicaments, la pratique de la médecine à domicile et l'administration des soins dans les hôpitaux ${ }^{63}$. Mais tout cela ne suffisait pas à ouvrir l'accès à la notabilité. Ce dernier objectif appelait un autre type de démarches et de stratégies déployées à l'échelle non plus des relations personnelles mais de la structure sociale elle-même.

\section{4 - LA RECONNAISSANCE SOCIALE}

Les comportements visés ici relèvent de trois sphères d'affirmation: la construction d'une identité corporative, l'intégration formelle à la structure des élites, l'accès à la respectabilité par le biais de fonctions civiques et honorifiques. La première sphère est celle de l'institutionnalisation du corps médical, dans le but de lui conférer une existence officielle et d'en faire un acteur collectif. C'était évidemment le rôle dévolu à la Société lors de sa création en 1900, les médecins saguenayens s'inspirant en cela d'un modèle bien connu à l'époque. En effet, des sociétés médicales virent le jour dans la plupart

63. Normand Perron, Un siècle de vie hospitalière au Québec..., 439; «Pour la reconnaissance de la profession de médecin, 1900-1930», Saguenayensia, (juillet-septembre 1984): 86-89. Nous possédons très peu de données sur ce dernier point. Mais on sait que les disputes de pouvoir entre les médecins et les administrations hospitalières étaient monnaie courante au Québec (par exemple: François Rousseau, La croix et le scalpel. Histoire des Augustines et de l'Hôtel-Dieu de Québec, II: $1892-1989$ (Québec, Les Éditions du Septentrion, 1994), 488 p.; François Guérard, «Les principaux intervenants dans l'évolution du système hospitalier en Mauricie, 1880-1939», RHAF, 48,3 (hiver 1995): 375-401; D. Goulet, F. Hudon et O. Keel, Histoire de l'Hôpital Notre-Dame de Montréal 1880-1980 (Montréal, VLB éditeur, coll. «Études québécoises», 1993), $458 \mathrm{p}$. 
des régions («districts») du Québec ${ }^{64}$. Chacune déléguait un gouverneur au Collège des médecins et chirurgiens. Au Saguenay, l'organisme tenait des réunions en principe trois fois par année en différents endroits de la région. À ces réunions, les médecins étaient soucieux de manifester leur présence à la population locale. En général, le protocole prévoyait une réception officielle organisée par les autorités municipales ainsi qu'une apparition à la messe, soulignée d'une mention par le curé en chaire. Les médecins appréciaient au plus haut point l'attention dont ils étaient alors l'objet. Leur image comme corps social (comme «classe», selon le mot employé dans les procèsverbaux) était un objet constant de préoccupations. Dans cet esprit, à la $65^{\mathrm{e}}$ réunion tenue le 20 octobre 1925, ils décidèrent que désormais, au décès d'un confrère, la Société ferait chanter des messes dans la paroisse du défunt, «afin que les gens se rendent bien compte de notre esprit de confraternité». Les confrères étaient priés de se faire un devoir d'assister à ces cérémonies. Les procès-verbaux reviennent souvent sur le sujet: les médecins se percevaient comme une «classe» distincte et ils entendaient bien affirmer en toute occasion leur identité.

Cette préoccupation se manifestait par la défense de leurs intérêts professionnels, auxquels les assemblées consacraient beaucoup de temps: lutte contre les praticiens illégaux, politique de tarification, vente non contrôlée de remèdes, prises de position sur divers projets de loi, etc. Les médecins veillaient aussi à mettre à jour leur savoir, à maîtriser les nouvelles techniques. À cette fin, les réunions comportaient ordinairement une partie scientifique au cours de laquelle on entendait un conférencier de l'extérieur ou un confrère à qui on avait demandé une intervention sur une matière relevant de la pratique courante. Les sujets les plus variés étaient alors abordés: l'emploi de la quinine dans les accouchements, la modulation des traitements en fonction du tempérament des patients, la mort apparente et la mort réelle, l'administration du chloroforme, l'application des mesures d'hygiène à la campagne, la vaccination contre la variole, etc. Les conférenciers de l'extérieur intervenaient souvent dans le cadre de programmes de «perfectionnement» gérés par des instances québécoises ou canadiennes ${ }^{65}$.

64. Les seules informations que nous possédions à ce sujet proviennent des procèsverbaux de la Société du Saguenay. Pour le Québec, voir D. Goulet, A. Paradis, Trois siècles d'histoire médicale au Québec..., section III.

65. Collège des médecins et chirurgiens, Fédération des Sociétés médicales, Conseil médical du Canada, Association médicale canadienne... 
Cela dit, au-delà des cadres et activités officielles et protocolaires, le fonctionnement de la Société connaissait bien des ratés. Les conférenciers locaux déclaraient souvent forfait; l'assistance aux réunions était ordinairement faible (plaintes récurrentes à ce sujet dans les procès-verbaux); un nombre important de médecins ne donnaient pas suite aux recommandations et directives régulièrement émises par la Société; des mésententes survenaient fréquemment quant au lieu des réunions; on avait du mal à faire payer la contribution annuelle donnant le statut de membre et, enfin, aux réunions, les divertissements l'emportaient très souvent sur la partie scientifique. On s'assemblait la plupart du temps dans des hôtels, parfois aussi dans des chalets, et on commençait par un repas copieux et bien arrosé qui ne préparait guère les esprits à percer les arcanes de l'art médical. De temps à autre, les procès-verbaux déplorent le désordre (le «manque de décorum») qui règne dans les assemblées. En 1938, on en vint à interdire l'alcool durant la conférence, résolution qui provoqua un «tintamarre». Tous ces traits révèlent l'individualisme du médecin en même temps que le caractère utilitaire de la Société. Celle-ci avait pour fonction de donner une image officielle du corps médical comme organisme responsable voué à servir la population. Elle servait égalemente d'instrument de représentation pour les médecins eux-mêmes. Cela étant acquis, la vie interne, les coulisses de l'organisme importaient finalement assez peu. Le visage social et la continuité de l'institution suppléaient à l'individualisme et à l'indiscipline de ses membres.

La deuxième sphère d'affirmation relève de tractations un peu surprenantes qui visaient à insérer formellement le corps médical dans la structure des élites régionales. L'affaire remonte à une assemblée de la Société tenue le 11 février 1915 au cours de laquelle les membres déploraient «l'apathie de la classe instruite» - et en particulier de certains ecclésiastiques - à l'endroit des médecins. En termes plus clairs, on croit comprendre que les médecins se plaignaient de ce que les autres professions libérales ne les considéraient pas vraiment comme leurs égaux ou leurs pairs, d'où la nécessité d'une intervention au plus haut niveau. Sous prétexte de déplorer les divisions au sein de la «classe instruite», le procès-verbal de la réunion énonce que:

[Les médecins] en souffrent, nos clients en souffrent quelquefois et nos curés le regrettent sincèrement. Pourquoi ne pas chercher à rapprocher davantage les gens de la classe instruite: Prêtres, avocats ou médecins, etc, qui tous ont une certaine somme d'influence séparément et qui en auraient bien davantage s'il y avait plus d'entente entre eux. Les relations les plus intimes réunissaient ces gens sur les bancs du collège. Pourquoi ces relations sociales ne se continuent-elles pas plus tard dans la vie, chacun 
travaillant dans sa sphère propre tout en respectant le travail des autres et s'unissant ensemble pour le bien commun.

Ce curieux préambule amène l'adoption d'une résolution en vertu de laquelle des membres de la Société,

[...] après étude des relations sociales actuellement existantes entre gens de la classe instruite, regrettent que ces relations soient plutôt nulles sinon hostiles, surtout entre gens de différentes professions et quelquefois même entre membres du clergé et les laïques. Qu'un tel état de choses est contraire à toutes les lois divines et humaines et de nature à enrayer tout mouvement tendant à améliorer notre situation religieuse, civile ou morale. Qu'en vue de prévenir les maux que pourrait engendrer une telle situation, une réaction s'impose et devrait partir de la classe dirigeante. C'est pourquoi la Société médicale invite respectueusement sa grandeur Mgr Labrecque, évêque de Chicoutimi, et tous les magistrats civils à étudier sérieusement la situation actuelle et à voir aux moyens propres à prendre pour l'améliorer, la Société médicale étant prête à endosser tout mouvement qui se fera en vue du bien commun.

Dans toute sa naïveté, la démarche était on ne peut plus transparente. Sous prétexte de cohésion sociale et de bien commun, les médecins entendaient bien intégrer le peloton des élites et être traités en conséquence dans les affaires de la collectivité. Dans ce but, ils recherchaient le patronage de la plus haute autorité religieuse, donnée ici comme gestionnaire de la structure du pouvoir régional. Pas de tous les pouvoirs sans doute, mais celui dans l'ordre duquel le corps médical désirait d'abord s'inscrire, en l'occurrence la hiérarchie socioculturelle, la notabilité, le reste venant ensuite. Après cette réunion, un document fut élaboré et présenté à l'évêque du diocèse le 21 décembre 1915. $\mathrm{M}^{\mathrm{gr}}$ Labrecque retint surtout l'idée d'unir sous sa houlette les différentes catégories sociales, la jugea tout à fait vertueuse et l'approuva. Du même coup, et sans être ignorant de la stratégie du corps médical, il le mandatait implicitement à poursuivre son projet et, surtout, lui conférait l'accréditation tant recherchée ${ }^{66}$.

La dernière sphère d'action - en toute logique avec ce qui précède - relève de comportements symboliques par lesquels les médecins revendiquaient et confirmaient à la fois leur appartenance à la notabilité et leur accès au sommet de la hiérarchie du prestige, de la respectabilité, de la moralité publique. Les médecins se devaient, en

66. Archives de l'évêché de Chicoutimi, Registre des lettres, lettre du 23 décembre 1915 adressée au docteur Adélard Riverin, alors secrétaire de la Société médicale. 
effet, d'être associés à toutes les manifestations, cérémonies et conduites mettant en cause ou célébrant les vertus civiques. À partir de 1920-1930, la grande majorité d'entre eux - surtout à la ville étaient propriétaires d'une maison bourgeoise, ce qui était le signe du bon citoyen jouissant paisiblement des fruits d'un travail honnête. Pères de famille nombreuse (supra), ils contribuaient comme tout le monde à l'expansion de la «race». Ils avaient des «manières», s'exprimaient «en termes», faisaient preuve de distinction et de raffinement, comme il convient à la «classe supérieure». À Chicoutimi, ils organisaient des thés où l'on causait, des soirées où l'on déclamait, des Euchre très animés dont la presse locale rendait compte avec force détails ${ }^{67}$.

De même, dans la vie publique, le médecin se faisait l'ambassadeur de toutes les vertus, l'artisan de toutes les bonnes causes. Il présidait les organismes de bienfaisance, occupait les premières places dans les grandes cérémonies religieuses, les congrès eucharistiques, les œuvres diverses en faveur de l'Église; il était aux premières loges dans les célébrations patriotiques et nationales: fête de la Saint-Jean, Rapatriement, colonisation... On le retrouvait aussi en politique municipale, dans des charges honorifiques (présidences de comités de fêtes, comités d'accueil, délégations officielles, patronage de fanfares, de milice volontaire, d'œuvres de jeunesse) et dans les institutions d'intérêt public: commissions scolaires, caisses populaires, commissions de crédit. Enfin, le corps médical était omniprésent dans la vie culturelle: organisation de conférences, de soirées de théâtre ou d'opéra, de réunions littéraires... Dans toutes ces occasions, le médecin affichait sa rectitude, sa prestance, son personnage hors du lot et se comportait à tous égards en «monsieur».

Le profit qu'il retirait de cette reconnaissance sociale allait bien au-delà du prestige ou de la considération dont jouissait un homme en vue; c'est la profession médicale elle-même qui se trouvait du même coup sanctionnée auprès de la population. Le corps médical avait ainsi les coudées franches pour étendre son influence dans le domaine des soins de santé et tenter de le gérer à sa guise. Par ailleurs, entre 1900 et 1940, la condition matérielle du médecin ne cessa de s'améliorer et il compta bien vite parmi les privilégiés, comme le montre une statistique de la richesse foncière, tirée d'un corpus de rôles d'évaluation $^{68}$. Aussi bien dans les campagnes que dans les villes, le

67. Selon Le Progrès du Saguenay du 31 janvier 1905, 5, quelques fillettes dansèrent un charmant menuet à une soirée donnée par le docteur Savard.

68. Il s'agit de 26 rôles d'évaluation foncière relatifs à 19 municipalités rurales et urbaines du Saguenay et couvrant la période 1879-1949. Tous ces rôles ont été informatisés et jumelés au fichier de population BALSAC, géré par l'IREP. 
médecin se situait toujours largement au-dessus de la moyenne dans l'échelle de la propriété foncière (tableau 1). Il est assuré que cette aisance faisait partie de l'image du notable et qu'elle a contribué à imposer le médecin comme personnage de marque. Mais on aurait tort d'accorder trop d'importance à cet attribut comme facteur de promotion et comme source du pouvoir ou de l'autorité que le médecin est parvenu à exercer dans cette société. Car dans ce cas particulier, la richesse peut aussi être interprétée comme le résultat d'une intégration réussie sur le plan social et culturel. C'est précisément son accréditation auprès de la population qui, en définitive, permettait au médecin de hausser des honoraires qui, dans la très grande majorité des cas, constituaient l'unique source de ses revenus ${ }^{69}$.

TABLEAU 1

Richesse foncière des médecins du Saguenay

1879-1949

\begin{tabular}{||l|c|c||}
\hline \multicolumn{1}{|c|}{$\begin{array}{c}\text { Groupes } \\
\text { socioprofessionnels }\end{array}$} & \multicolumn{2}{c|}{ Évaluation moyenne (\$) } \\
\cline { 2 - 3 } & $\mathrm{N}$ & Valeur \\
\hline Cultivateurs & 3933 & 2279 \\
Ouvriers semi et non qualifiés & 2054 & 1467 \\
Ouvriers qualifiés & 147 & 2575 \\
Artisans & 960 & 2249 \\
Travailleurs non manuels semi et non qualifiés & 88 & 2324 \\
Travailleurs non manuels qualifiés & 180 & 3003 \\
Notables & $1019^{1}$ & 3918 \\
\hline TOTAL & 8381 & 2297 \\
\hline MÉDECINS & 43 & 5658 \\
\hline
\end{tabular}

1. Le nombre de Notables paraît élevé. Ce groupe inclut une proportion importante d'individus se déclarant marchands ou entrepreneurs.

Source: Échantillon de 26 rôles d'évaluation foncière relatifs à 19 municipalités urbaines et rurales. IREP.

69. Durant l'époque considérée, le modèle du médecin/homme d'affaires n'existait guère au Saguenay, si on excepte l'exploitation de pharmacies et certains investissements dans l'immobilier qui canalisaient une partie de l'épargne. Cet énoncé s'appuie sur les données orales et sur la lecture des journaux régionaux entre 1890 et 1930. 
La tradition orale et l'historiographie saguenayennes ont perpétué le souvenir de certains médecins qui semblent avoir incarné au plus haut point cette éthique de la distinction et qui ont ainsi contribué à nourrir le stéréotype du médecin-notable. C'est le cas, par exemple, de Louis-Élie Beauchamp de Chicoutimi, qui fit un beau mariage avec une jeune fille du «grand monde»; il fut un bienfaiteur de l'Église, chantre dans la chorale de la cathédrale et maire de Chicoutimi. À Hébertville, le docteur Casgrain était un vrai «seigneur» (c'est ainsi qu'on l'appelait du reste), tout en finesse, et qui formait avec sa femme un couple des plus élégants. À Roberval, le docteur Constantin était maire lui aussi et, en plus, écrivain et musicien, homme de cœur et d'esprit... Toutes les régions du Québec ont connu cet «idealtypus». On pense ici, parmi tant d'autres, au célèbre docteur Fortin de Gaspé, officier de milice, magistrat et sénateur ${ }^{70}$. Et on pense surtout au docteur Joseph Gauvreau ${ }^{71}$, qui a en quelque sorte tracé le portrait du médecin idéal qu'il a voulu lui-même incarner: ami, compagnon dans la souffrance, il est un parfait psychologue et «un moraliste ferme dans ses principes». Comme le prêtre, il exerce un sacerdoce. Ses qualités indispensables sont: «la bonne tenue, la distinction des manières, la maîtrise de soi, la réserve, le tact, la fierté». Il cultive les qualités de l'esprit, fréquente régulièrement les retraites fermées; il est irréprochable en tout ${ }^{72}$. Malheureusement, selon le docteur Gauvreau, tous ces traits seraient désormais en péril par la faute des patients qui, de plus en plus, ne songeraient qu'à guérir à tout prix et n'exigeraient rien d'autre du médecin...

\section{CONCLUSION}

Cette dernière référence illustre parfaitement l'hypothèse défendue dans le présent essai. Il est certain que l'insertion et l'ascension sociales du médecin devaient beaucoup à son savoir, à sa compétence ${ }^{73}$. Elles étaient servies aussi par des stratégies habiles de com-

70. Damase Potvin, Le roi du golfe. Le Docteur P.-E. Fortin, 1823-1888, ancien commandant de la Canadienne (Québec, Éditions du Quartier Latin, 1952), $181 \mathrm{p}$.

71. Joseph Gauvreau, Les médecins au Canada français: vade-mecum de l'étudiant en médecine et du jeune médecin (Montréal, Ducharme, 1933), $116 \mathrm{p}$.

72. On retrouvait plusieurs de ces traits chez James Miles Langstaff, ce médecin rural ontarien dont la pratique professionnelle a pu être reconstituée en détail par Jacalyn Duffin, Langstaff: a Nineteenth Century Medical Life (Toronto, University of Toronto Press, 1993), $383 \mathrm{p}$.

73. On ne doit pas sous-estimer, de ce point de vue, l'importance des progrès réalisés durant la première moitié du $\mathrm{XX}^{\mathrm{e}}$ siècle: rayons $\mathrm{X}$, meilleur contrôle des infections post-opératoires, amélioration des mesures d'hygiène publique. François Guérard, La santé publique dans deux villes du Québec de 1887 à 1939: Trois-Rivières et Shawinigan, thèse de doctorat (histoire), Université du Québec à Montréal, 1993, 525 p.; D. Goulet, A. Paradis, Trois siècles d'histoire médicale au Québec... 
promis avec la médecine populaire et de mise en dépendance des familles. Mais au-delà du métier ou de la stricte fonctionnalité, le poids des interférences sociales et culturelles était considérable. Pourquoi donc fallait-il que le bon médecin se caractérise également par des manières distinguées, par un esprit cultivé, par d'éminentes qualités civiques? Et pourquoi les médecins du Saguenay ont-ils éprouvé le besoin de s'assurer le haut patronage de l'évêque? Cette démarche, en particulier, révèle clairement la position et l'orientation collective du corps médical, en même temps que le type d'autorité qu'il entendait exercer. Toute son action s'inscrivait en priorité dans la hiérarchie socioculturelle, mais d'une manière stratégique: c'est son accréditation dans la notabilité qui lui garantissait la liberté d'action professionnelle dont il avait besoin pour établir son monopole dans les soins de santé, pour ajuster régulièrement son échelle de tarification et pour s'ériger en égal auprès des autres professions libérales ${ }^{74}$.

L'action du corps médical éclaire aussi la structure de la notabilité, indiscutablement dominée par le clergé qui était explicitement mandaté pour en arbitrer l'accès. On relève, à cet égard, un échange de bons procédés. D'un côté, les médecins tiraient un avantage considérable à l'aval que lui octroyait le haut clergé. Mais ce dernier trouvait aussi son profit dans l'opération. Les médecins devenaient, en effet, des alliés quil, dans l'arène sociale, culturelle et politique, allaient agir dans le cadre tracé par l'Église et ainsi fortifier ses positions. Ces remarques vont dans le sens d'une mutualité d'intérêts et d'une alliance étroite entre les élites socioculturelles, sous la gouverne du clergé ${ }^{75}$. Mais on ne tient là qu'une facette de la dynamique du pouvoir dans ce genre de société dont l'économie était largement possédée et dirigée de l'extérieur. Le grand capital nord-américain (et même européen) contrôlait à distance la structure de la production industrielle, ce qui créait localement un espace pour un pouvoir social et culturel. L'histoire des relations entre ces deux lieux de pouvoir

74. Dans ces conditions, la promotion sociale du médecin apparaît comme un processus complexe à plusieurs volets, qui a trouvé des appuis à l'échelle à la fois microsociale et sociétale. Pour cette raison, on doit se méfier un peu de certaines thèses simplificatrices comme celle de R. Hamowy, Canadian Medicine, a Study in Restricted Entry (Vancouver et Toronto, The Fraser Institute, 1984), qui fait tout reposer sur la poursuite égoïste d'intérêts économiques, servie par des stratégies manipulatrices. Rappelons à ce propos que, dans sa recherche sur l'évolution de la médecine québécoise entre 1788 et 1909, J. Bernier, La médecine au Québec..., a lui aussi fait ressortir l'importance des facteurs sociaux et culturels dans la redéfinition de la condition du médecin. R. D. Gidney et W. P. J. Millar, Professional Gentlemen: the Professions..., chapitre 5, ont proposé le même modèle dans le contexte ontarien.

75. Sur ce point, nous sommes en désaccord avec Gérald Fortin, «Le nationalisme canadien-français et les classes sociales», RHAF, 22,4 (mars 1969): 525-540, pour qui le clergé, berné par leur stratégie, se serait en quelque sorte laissé exploiter par les professions libérales. 
relève d'un autre type d'analyse. Ce qu'on en sait d'ores et déjàa ${ }^{76}$ donne à penser que: a) ces rapports n'étaient pas toujours harmonieux mais reposaient, pour l'essentiel, sur une interdépendance bien comprise de part et d'autre; b) du côté du pouvoir local, ils étaient gérés par le haut clergé, qui servait d'interface avec les représentants de la finance et de l'industrie; c) ils étaient en définitive («en dernière instance») dominés par le grand capital ${ }^{77}$. Ces données structurelles doivent de toute évidence être prises en compte pour situer correctement l'aire de pouvoir impartie aux élites locales.

76. Voir notamment José E. Igartua, «'Corporate' Strategy and Locational DecisionMaking: the Duke-Price Alcoa Merger, 1925», Journal of Canadian Studies/Revue d'études canadiennes, 20,3 (automne 1985): 82-101; José E. Igartua, Arvida au Saguenay: naissance d'une ville industrielle (ouvrage à paraître chez McGill-Queen's University Press, 1995); Gaston Gagnon, Un pays neuf. Le Saguenay-Lac-Saint-Jean en évolution. Alma (Les Éditions du Royaume, 1988), 196 p.; Gérard Bouchard, «Introduction à l'étude de la société saguenayenne aux $\mathrm{XIX}^{\mathrm{e}}$ et $\mathrm{XX}^{\mathrm{e}}$ siècles», RHAF, 31,1 (juin 1977): 3-27; Gérard Bouchard, «Les prêtres, les capitalistes et les ouvriers à Chicoutimi (1896-1930)», Mouvement social, 112 (juillet-septembre 1980): 6-23.

77. Gérard Bouchard, «Introduction à l'étude de la société...», 3-27. 